\title{
Foamy Virus Vectors
}

\author{
DAVID W. RUSSELL ${ }^{1,2 *}$ AND A. DUSTY MILLER ${ }^{1,3}$ \\ Fred Hutchinson Cancer Research Center, Seattle, Washington 98104, ${ }^{1}$ and Division of Hematology ${ }^{2}$ and \\ Department of Pathology, ${ }^{3}$ University of Washington, Seattle, Washington 98195
}

Received 20 July 1995/Accepted 28 September 1995

\begin{abstract}
Human foamy virus (HFV) is a retrovirus of the spumavirus family. We have constructed vectors based on HFV that encode neomycin phosphotransferase and alkaline phosphatase. These vectors are able to transduce a wide variety of vertebrate cells by integration of the vector genome. Unlike vectors based on murine leukemia virus, HFV vectors are not inactivated by human serum, and they transduce stationary-phase cultures more efficiently than murine leukemia virus vectors. These properties, as well as their large packaging capacity, make HFV vectors promising gene transfer vehicles.
\end{abstract}

The three major subfamilies of retroviruses are oncoviruses, such as murine leukemia virus (MLV); lentiviruses, such as human immunodeficiency virus (HIV); and foamy viruses or spumaviruses (51). Foamy viruses have been isolated from many vertebrate species, including primates, and are not known to cause disease (14), even in accidentally infected research personnel (47). Previous reports of human diseases associated with foamy virus infection have not been confirmed by an extensive analysis of the prevalence of foamy viruses in human populations (47). Human foamy virus (HFV) was originally derived from a human nasopharyngeal carcinoma cell line (1); however, subsequent studies indicate that it may be a chimpanzee virus variant $(3,47)$.

Foamy viruses have the largest genome size of all the retroviruses (9). The HFV genomic RNA is 11,021 nucleotides in length and, in addition to the gag, pol, and env genes found in all retroviruses, contains three open reading frames (bell to bel3) downstream from the env gene that are unique to spumaviruses $(9,10,25)$ (see Fig. 1). Multiple splicing patterns predict that as many as six gene products are encoded by the bel reading frames (32), one of which (Bel1) is a transactivator of transcription from the viral long terminal repeat (LTR) promoter $(17,40,49)$. The HFV genome also contains a second promoter in the env gene that directs transcription of the bell gene early in infection $(20,21)$.

HFV has been cloned as an infectious provirus $(22,39)$, and a recent report describes the construction of replicating foamy virus vectors with deletions in the bel2-3 region (45). We have constructed replication-incompetent transducing vectors by replacing a portion of the env gene and bell promoter with reporter genes. These vectors can be packaged as viral particles by cotransfection with helper plasmids. In this work, we have studied the transducing properties of HFV vectors and compared them with vectors based on MLV. Our results suggest that HFV vectors offer several potential advantages over currently available viral vectors and that they may prove useful for a wide variety of gene transfer applications.

\section{MATERIALS AND METHODS}

Cell culture. The cultured cells used included normal human foreskin fibroblasts (34), human 293 cells (11), COS-7 cells (simian virus 40 [SV40]-transformed African green monkey kidney cells; ATCC CRL 1651), Vero African

\footnotetext{
* Corresponding author. Present address: Markey Molecular Med-
} icine Center, Mailstop 357720, University of Washington, Seattle, WA 98195-7720. Phone: (206) 616-4562. Fax: (206) 543-3560. green monkey kidney cells (ATCC CCL 81), MDBK bovine kidney cells (ATCC CCL 22), sheep choroid plexus cells (ATCC CRL 1700), Cf2Th canine thymus cells (ATCC CRL 1430), CCC-81 cat cells transformed with Moloney murine sarcoma virus (8), AH 927 cat cells (from J. Overbaugh; originally from W. Nelson-Rees), NRK normal rat kidney cells (6), 208F rat fibroblasts (37), NIH $3 \mathrm{~T} 3 \mathrm{TK}^{-}$mouse fibroblasts (50), PA317 mouse amphotropic MLV vector packaging cells (28), CHO-K1 Chinese hamster ovary cells (16), BHK21 baby hamster kidney cells (24), FAB hamster HFV $\beta$-galactosidase indicator cells (53), and primary White Leghorn chicken embryo fibroblasts (from P. Neiman). Unless otherwise indicated, all cells were grown in Dulbecco's modified Eagle's medium (DMEM) with $10 \%$ fetal bovine serum (FBS) at $37^{\circ} \mathrm{C}$ in $10 \% \mathrm{CO}_{2}$. FBS was heat inactivated for $30 \mathrm{~min}$ at $56^{\circ} \mathrm{C}$ except as indicated in the legend to Fig. 5 . Nonessential amino acids were added to CHO-K1 cultures.

Stationary-phase $208 \mathrm{~F}$ and human fibroblast cultures were prepared by maintaining confluent cultures for 2 to 3 weeks in DMEM containing 5\% FBS and $10^{-6} \mathrm{M}$ dexamethasone as described before $(30,43)$. Dividing cultures were prepared by treating them with trypsin and plating the cells at a density of either $2.5 \times 10^{5}$ cells per 35 -mm well in six-well plates or $4 \times 10^{5}$ cells per 60 -mm dish the day before infection. The percentage of cells passing through $\mathrm{S}$ phase every $24 \mathrm{~h}$ was determined by autoradiography (43).

Vector construction and production. Relevant restriction sites are shown in Fig. 1. pFdelGP is a deletion derivative of pHSRV13 (22) lacking the internal NcoI fragment. pFGPXhoI (not shown) is a deletion derivative of pHSRV13 made by digestion with $B s p E I$ and $B p u$ 1102, end-filling with the Klenow fragment of DNA polymerase I, and insertion of an XhoI linker (5'-CCTCGAGG$\left.3^{\prime}\right)$. pFGPSN was made by inserting an NheI-XhoI fragment of pLXSN (29) containing the SV40 early promoter and neomycin phosphotransferase gene into the $X h o I$ site of pFGPXhoI. pFGPMAP was made by inserting a Sall-BspMI fragment of pALAPSN (43) containing the MLV LTR promoter and the human placental alkaline phosphatase gene into the XhoI site of pFGPXhoI.

Vector stocks were made by calcium phosphate cotransfection of BHK21 cells with $10 \mu \mathrm{g}$ each of vector plasmid and helper plasmid per 6-cm dish, further culture for 3 to 11 days, with medium changes every 2 to 3 days, collection of culture medium, and filtration through cellulose acetate filters $(0.45-\mu \mathrm{m}$ pore size). Stocks were stored in aliquots at $-76^{\circ} \mathrm{C}$. Unless otherwise indicated, stocks were prepared by transfecting with pFGPMAP and pHSRV13 (FGPMAP) or pFGPSN and pFdelGP (FGPSN). Stocks of the MLV vector LAPSN (31) were prepared in PA317 cells as described before (29).

Transduction assays. Unless otherwise indicated, all cells were plated at $2 \times$ $10^{5}$ cells per $35-\mathrm{mm}$ well the day before infection, and infections were performed by adding dilutions of vector stocks in the presence of Polybrene $(4 \mu \mathrm{g} / \mathrm{ml}$; Sigma). Polybrene did not increase the transduction efficiency of HFV vectors (data not shown) but was included to improve transduction of control cultures by MLV vectors. Transduction of G418 resistance was performed by infecting on day 1 , treating with trypsin, plating the cells at different dilutions in DMEM with $10 \%$ FBS on day 2, and adding active G418 to $0.50 \mathrm{mg} / \mathrm{ml}$ (human fibroblasts) or $0.70 \mathrm{mg} / \mathrm{ml}$ (FAB cells and 208F cells) on day 3 (human fibroblasts and $208 \mathrm{~F}$ cells) or day 2 (FAB cells). Surviving colonies were counted after staining with Coomassie brilliant blue $\mathrm{G}$ on days 7 to 10 , when all cells in control cultures were dead. Transduction of alkaline phosphatase was measured by histochemical staining 2 days after infection and counting individual stained foci of cells (7). Helper virus expressing the bel1 gene was assayed in parallel with vector assays by staining FAB cells for $\beta$-galactosidase 2 days after infection (53).

\section{RESULTS}

Production of foamy virus vectors. We replaced a portion of the env gene of the pHSRV13 HFV provirus (22) with either 


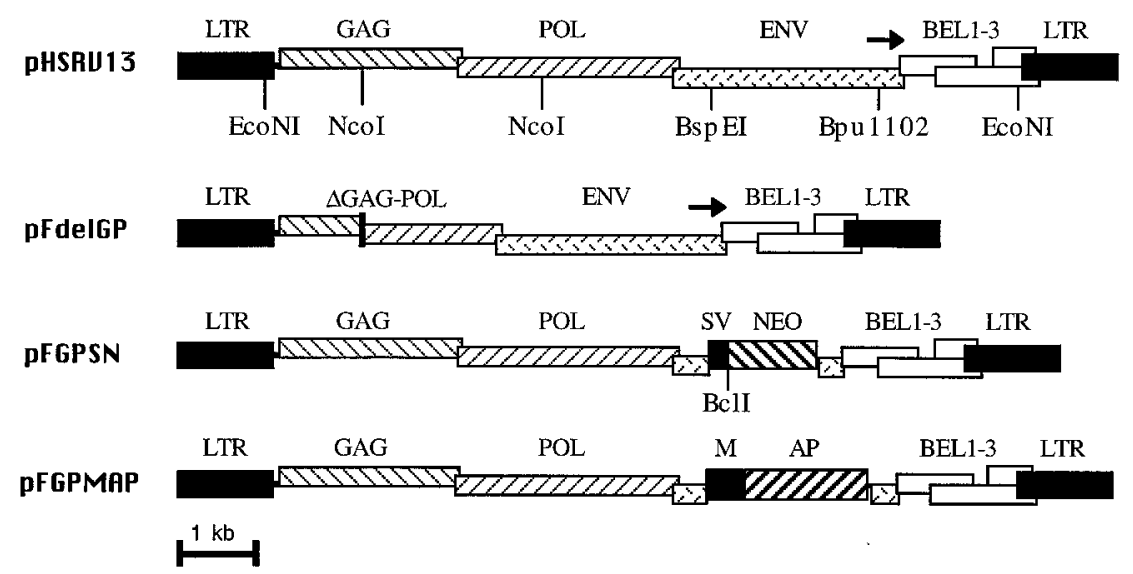

FIG. 1. Foamy virus constructs. The structures of HFV constructs used to generate vector stocks are shown. The positions of the viral gag, pol, env, and bel genes are indicated, as are the HFV LTRs and the internal viral promoter (arrow). Also shown are the pFdelGP gag-pol deletion and the SV40 early promoter (SV), neomycin phosphotransferase gene (NEO), MLV LTR promoter (M), and alkaline phosphatase gene (AP) present in the pFGPSN and pFGPMAP vectors. The locations of relevant restriction sites are shown below the constructs.

the neomycin phosphotransferase gene under the control of the SV40 early promoter or the human placental alkaline phosphatase gene under the control of the MLV LTR promoter to make the vector constructs $\mathrm{pFGPSN}$ and $\mathrm{pFGPMAP}$, respectively (Fig. 1). This env deletion prevents the synthesis of a functional envelope protein and also disrupts the internal bell promoter required for high-level expression of the Bel1 transactivator protein. Vector stocks were generated by cotransfection of BHK21 cells with a vector plasmid construct and either the wild-type pHSRV13 plasmid or the pFdelGP helper construct, containing a deletion in the gag and pol genes (Fig. 1).

Functional vector particles were measured by infecting FAB cells with filtered culture medium collected at different times after transfection. FAB cells contain an integrated copy of a nuclear $\beta$-galactosidase gene under the control of the HFV LTR promoter (53), so infection by viruses expressing the bel1 gene leads to transactivation of the HFV LTR, the production of $\beta$-galactosidase, and blue histochemical nuclear staining. As the $\mathrm{pFGPSN}$ and $\mathrm{pFGPMAP}$ vector constructs do not produce high levels of Bel1, infection by vector particles does not lead to detectable $\beta$-galactosidase expression, and transduction can be measured by G418 resistance (FGPSN) or purple histochemical staining for alkaline phosphatase (FGPMAP). Although both titering methods measure gene expression from an internal promoter, there could be differences in titering efficiency as it relates to virion particle numbers.

After transfecting BHK21 cells with pFGPSN and pHSRV13, vector titers were approximately 10 -fold lower than helper virus titers, while transfection with pFGPSN and pFdelGP produced nearly equivalent vector and helper titers after 7 days (Fig. 2A and B). The higher helper titers obtained with pHSRV13 were not unexpected, as this construct produces replication-competent virus, while pFdelGP can only produce helper particles in the presence of gag and pol genes encoded by the vector construct. When BHK21 cells were transfected with pFGPSN and pHSRV13 and then cultured in the presence of G418 to ensure that all cells infected with helper virus also contain vector sequences, vector titers were as high as helper titers (Fig. 2C). With the same transfection procedures, the $\mathrm{pFGPMAP}$ construct produced similar titers, ranging from $1 \times 10^{3}$ to $5 \times 10^{4} \mathrm{FAB}$ cell transducing units at 5 to 7 days after transfection (data not shown). We have also produced vector stocks by transfecting NIH $3 \mathrm{~T} 3 \mathrm{TK}^{-}$cells, 293 cells, and COS-7 cells (data not shown).
Transduction of different cell types by foamy virus vectors. We screened a series of cell lines from different species to determine the range of cells that can be transduced by HFV vectors. Fourteen cell lines from species ranging from humans to chickens were all transduced by the FGPMAP vector (Table $1)$. The small differences in transduction efficiencies did not correlate with evolutionary divergence among species, cell histological type, or state of cellular transformation.

Integration of vector genomes. The structure of vector genomes present in transduced cells was determined by Southern analysis. Independent G418-resistant clones were isolated from rat $208 \mathrm{~F}$ cells infected with FGPSN and expanded to more than $10^{7}$ cells before high-molecular-weight DNA was isolated. Figure 3 shows the results of EcoNI and BclI restriction digests from six representative G418-resistant clones probed with neo sequences. EcoNI cleaves inside both terminal repeats of pFGPSN, producing an 8.9-kb fragment containing the FGPSN coding regions, while $B c l$ l cleaves $5^{\prime}$ to the neo gene, producing fragments with $3^{\prime} \mathrm{BclI}$ sites in adjoining chromosomal DNA (see Fig. 1). Three of these cell lines (clones 1 to 3 ) contained the EcoNI fragment expected from intact proviruses bounded by LTRs. Two transductants (clones 4 and 6) contained smaller proviruses, which further analysis showed contained the gag-pol deletion present in the pFdelGP helper construct (data not shown). We have analyzed a total of 10 transductants: 6 contained intact proviruses, 3 contained gagpol deletions from pFdelGP, and 1 (clone 5) contained a provirus with a larger than expected EcoNI fragment, which we have not characterized further (Fig. 3 and data not shown). Digestion with $B c l$ I produced various sizes of neo-hybridizing restriction fragments, consistent with random integration. No integrated helper virus was detected in any of the clones analyzed when the blots were probed with viral sequences (data not shown).

Effect of cell proliferation on transduction. We compared the transduction rates of HFV vectors on stationary-phase and dividing cultures of human fibroblasts and rat $208 \mathrm{~F}$ cells. Infections were performed at low multiplicities to avoid coinfection with helper virus. Stationary-phase cultures were prepared by maintaining confluent cell monolayers in $5 \%$ FBS and $10^{-6}$ $\mathrm{M}$ dexamethasone under conditions that resulted in approximately $4 \%$ (human fibroblasts) or $<1 \%$ (208F cells) of the cells passing through $\mathrm{S}$ phase each day $(30,43)$. Dividing cultures were subconfluent cells grown in $10 \%$ FBS, with $60 \%$ (human 

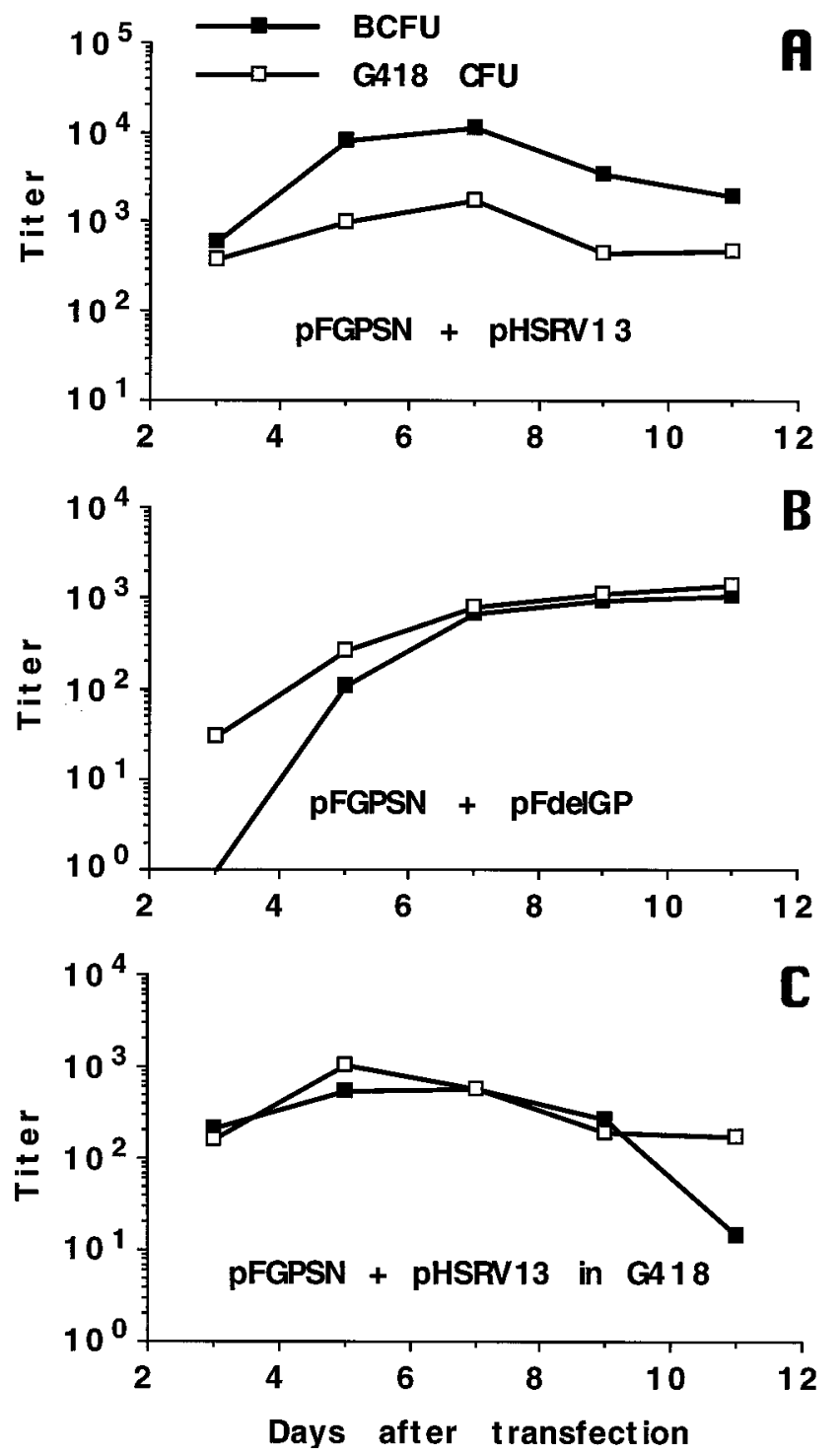

FIG. 2. Foamy virus vector production. BHK21 cells were cotransfected with pFGPSN and pHSRV13 (A and C) or pFdelGP (B) and then cultured for an additional 11 days. The medium was changed the day after transfection (day 1 ) and then collected and replaced every 2 days afterwards. Collected medium was filtered and used to infect FAB cells. Vector titers were measured by the production of G418-resistant colonies (G418 CFU), and helper titers were measured as blue-cell-forming units (BCFU) after staining for $\beta$-galactosidase. In panel B, the 3-day BCFU titer was $<1 / \mathrm{ml}$. In panel C, G418 ( $700 \mu \mathrm{g}$ of active drug per $\mathrm{ml}$ ) was added to the culture on day 2 . Titers are per milliliter.

fibroblasts) or $>90 \%$ (208F cells) of the cells passing through $\mathrm{S}$ phase each day (43) (data not shown). If transduction occurs only in proliferating cells, stationary-phase cultures should be transduced at approximately $7 \%$ or $<1 \%$ the rate of dividing cultures for human fibroblasts and $208 \mathrm{~F}$ cells, respectively.

Figure 4 shows the relative transduction efficiencies of HFV and MLV vectors on stationary-phase and dividing cultures, plotted as the stationary-to-dividing efficiency ratio ( $\mathrm{S} / \mathrm{D}$ ratio). In an alkaline phosphatase assay which can be performed without stimulating cultures to divide, the S/D ratio for HFV vectors was 0.18 on human fibroblast cultures and 0.07 on $208 \mathrm{~F}$ cells. While these results show that HFV vectors preferentially transduce proliferating cells, the ratios are higher than those expected if transduction occurred only in cells actively prolif-
TABLE 1. Transduction of different cell types by the HFV vector FGPMAP ${ }^{a}$

\begin{tabular}{|c|c|c|c|}
\hline Species & Cell type & $\mathrm{AP}(\mathrm{FFU} / \mathrm{ml})$ & $\begin{array}{l}\text { Relative transduction } \\
\text { efficiency }{ }^{b}\end{array}$ \\
\hline Human & Normal fibroblasts & 17,000 & 1.00 \\
\hline \multirow[t]{2}{*}{ Monkey } & COS-7 & 2,300 & 0.13 \\
\hline & Vero & 4,300 & 0.25 \\
\hline Steer & MDBK & 2,400 & 0.14 \\
\hline Sheep & Choroid plexus & 2,900 & 0.17 \\
\hline Dog & Cf2Th & 1,600 & 0.09 \\
\hline \multirow[t]{2}{*}{ Cat } & CCC-81 & 16,000 & 0.93 \\
\hline & AH 927 & 2,500 & 0.15 \\
\hline \multirow[t]{2}{*}{ Rat } & NRK & 9,200 & 0.54 \\
\hline & $208 \mathrm{~F}$ & 3,900 & 0.23 \\
\hline Mouse & NIH $3 \mathrm{~T} 3 \mathrm{TK}^{-}$ & 5,100 & 0.30 \\
\hline \multirow[t]{2}{*}{ Hamster } & CHO-K1 & 6,400 & 0.37 \\
\hline & FAB & 8,400 & 0.49 \\
\hline Chicken & Embryo fibroblasts & 2,700 & 0.16 \\
\hline
\end{tabular}

${ }^{a}$ Each cell type was infected with FGPMAP and stained for alkaline phosphatase (AP) expression 2 days later, and the number of AP focus-forming units (FFU) per milliliter (FFU/ml) of vector stock was determined. All results are the averages of two independent measurements that varied by less than $36 \%$ from the mean. No AP foci were observed in uninfected control cultures.

${ }^{b}$ Relative transduction efficiency is the AP FFU/ml on each cell type divided by the AP FFU/ml on normal human fibroblasts.

erating at the time of infection and higher than those obtained with MLV vectors. In a G418 resistance assay, which requires that cells be treated with trypsin after the infection and allowed to divide during selection, the $\mathrm{S} / \mathrm{D}$ ratio was 1.0 for $\mathrm{HFV}$ vectors and 0.14 for MLV vectors. The higher S/D ratios obtained with the G418 resistance assay suggest that efficient vector gene expression occurs after these cells are stimulated to divide.

Human serum does not inactivate HFV vectors. Human serum is known to inactivate several animal retroviruses, including MLV (52), while human retroviruses such as human T-cell leukemia virus and HIV are not inactivated by human serum, which may contribute to their pathogenicity $(2,15)$. We measured transduction by HFV and MLV vectors after incubating them with three different human serum samples. As shown in Fig. 5, there was no decrease in the transduction efficiency of FGPMAP after serum exposure under conditions that inactivated MLV vectors $(42,48)$. Thus, HFV is similar to

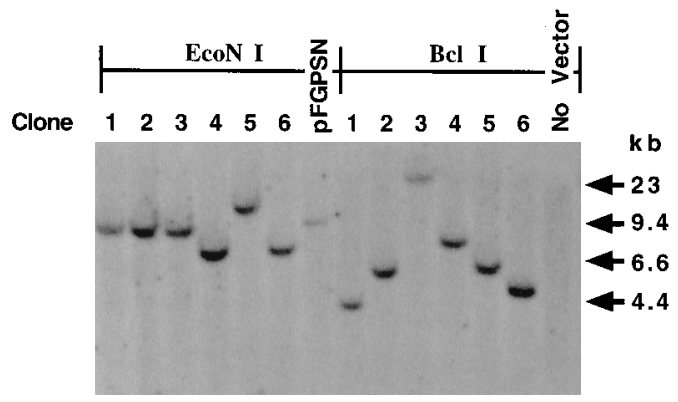

FIG. 3. Integration of foamy virus vector genomes. Southern analysis was performed with a neo gene probe and $10 \mu \mathrm{g}$ of high-molecular-weight DNA isolated from six independent G418-resistant clones of $208 \mathrm{~F}$ cells transduced by FGPSN (lanes 1 to 6 ) or untransduced $208 \mathrm{~F}$ cells (No Vector lane). The pFGPSN lane contains $10 \mathrm{pg}$ of pFGPSN plasmid DNA mixed with untransduced 208 F cell DNA. Samples were digested with $E c o$ NI or $B c l$ I as indicated. The positions of size standards from the same gel are shown. 


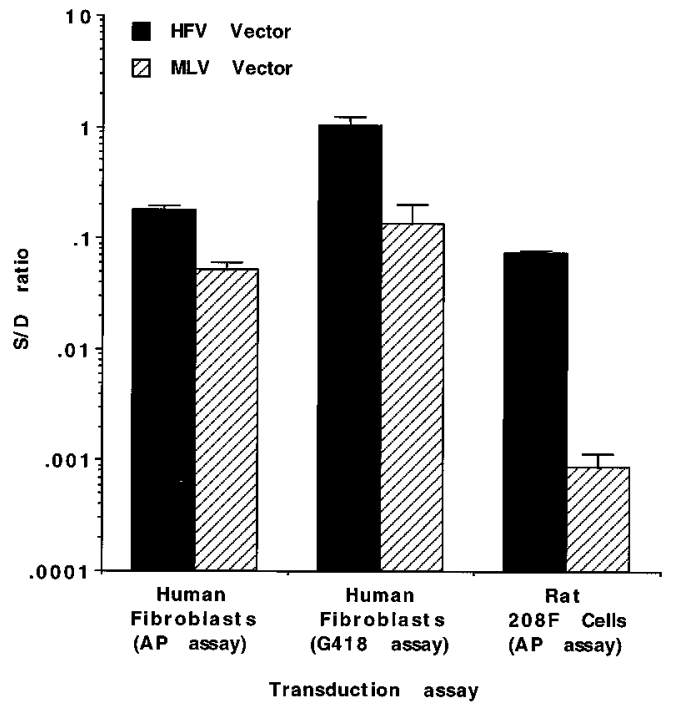

FIG. 4. Transduction in stationary-phase and dividing cultures. Stationaryphase and dividing cultures of human fibroblasts and rat $208 \mathrm{~F}$ cells were transduced with the HFV vector FGPMAP (solid columns, alkaline phosphatase [AP] assay), the HFV vector FGPSN (solid columns, G418 assay), and the MLV vector LAPSN produced in PA317 cells (hatched columns) and then stained for alkaline phosphatase expression (AP assay) or selected for G418 resistance (G418 assay). The S/D ratio is the vector titer measured in stationary-phase cultures divided by that in dividing cultures. Mean values are plotted with standard errors. FGPMAP infections contained less than one transducing particle and less than 10 helper particles per 1,000 cells. FGPSN infections contained less than one transducing particle and less than 0.1 helper particle per 5,000 cells.

known human retroviruses in its resistance to human complement.

\section{DISCUSSION}

Vector production. The transient cotransfection procedure that we have used generated vector stocks with titers as high as $5 \times 10^{4}$ per ml. Wild-type HFV can be grown to titers of up to $10^{9} / \mathrm{ml}$ under optimal conditions (53), so higher vector titers may be possible with improved packaging systems. Vector stocks free of helper virus were generated at early times after transfection by using the pFdelGP deleted helper construct (see Fig. 2B). However, a single recombination event between vector and helper constructs could have generated replicationcompetent helper virus at later time points. Further modifications of the helper construct should prevent the formation of replication-competent helper virus, as was the case for MLV packaging lines (27).

Host range. Every vertebrate cell type that we tested was transduced by HFV vectors, suggesting that the receptor used for viral entry is a common cell surface molecule. Previous studies concluded that some cell lines, including COS-7 cells, were resistant to HFV infection, based on the absence of observed cytopathic effects $(3,20,22)$ and decreased virus production (23). However, COS-7 cells were transduced by $\mathrm{HFV}$ vectors in our experiments. Thus, it is possible that virus entry, reverse transcription, and gene expression can occur without cytopathic effects (syncytium formation) or the production of functional viral particles in some cell types.

Provirus integration. Cultures infected with foamy viruses usually contain high copy numbers of episomal, linear cDNA molecules that may be generated by repeated infections (18, 26, 38). As these cultures are producing viral particles, the

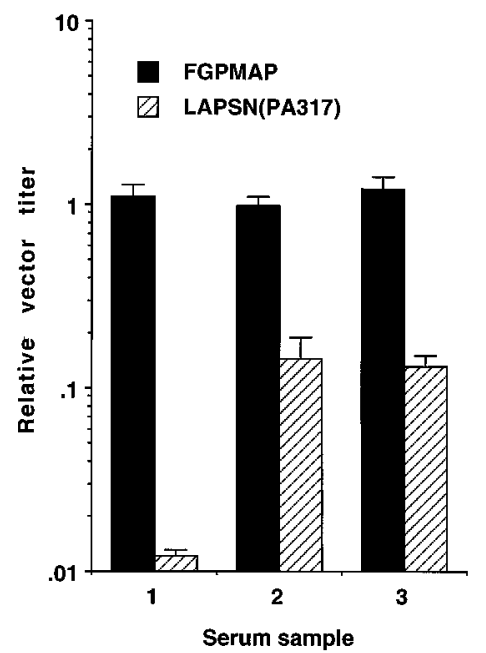

FIG. 5. HFV vectors are not inactivated by human serum. Stocks of FGPMAP and LAPSN(PA317) were incubated with an equal volume of serum from three different individuals for $30 \mathrm{~min}$ at $37^{\circ} \mathrm{C}$ and then used to transduce rat $208 \mathrm{~F}$ cells as described before (42). Results are shown as the titer of vector stocks incubated with human serum divided by the titer of stocks incubated under identical conditions with heat-inactivated serum $\left(30 \mathrm{~min}\right.$ at $\left.56^{\circ} \mathrm{C}\right)$. Mean values are plotted with standard errors. Heat-inactivated serum samples did not decrease vector titers compared with the titers after incubation with DMEM containing 10\% FBS (data not shown).

episomal viral DNA molecules could be transcriptionally active. Although foamy viruses contain reverse transcriptase and integrase genes that should allow the formation of an integrated provirus, the limited life span of actively infected cells and excess of episomal molecules make the detection of integrated proviruses problematic. Integrated simian foamy virus 3 genomes were detected in infected Vero cells grown for more than 50 passages in the presence of $3^{\prime}$-azido- $3^{\prime}$-deoxythymidine to prevent cytopathic effects (46). However, the efficiency of integration is not known, and the entire foamy virus life cycle could occur without chromosomal integration in most infected cells.

All of the G418-resistant transductants that we analyzed contained integrated vector proviruses, most of which appeared to be randomly integrated, intact vector genomes bounded by LTRs. Thus, foamy virus vectors integrate in a typical retroviral pattern. The deleted proviruses that we detected were presumably created by recombination between vector and helper sequences, which could have happened during the transfection procedure or during reverse transcription. Transductants contained single-copy proviruses and no helper genomes, so vector integration occurred in the absence of helper virus.

Effects of cell proliferation on transduction. Oncoviruses such as MLV require cell proliferation for efficient infection (13), while lentiviruses such as visna virus and HIV do not (12, 19). In the case of MLV, mitosis is required for nuclear entry of viral particles (41), and vectors based on MLV require cell division for efficient transduction (30). Although syncytium formation and virus production are decreased when confluent cultures are infected by foamy viruses $(23,35,36)$, it is not known if viral DNA synthesis and gene expression require cell proliferation. Our findings suggest that HFV vectors preferentially transduce dividing cultures, but the transduction efficiency on stationary-phase cultures is higher than for MLV vectors and higher than expected if transduction occurs only in cells proliferating at the time of infection. The HFV Gag 
protein contains a functional nuclear localization signal (44), so it is possible that nuclear entry of HFV particles is independent of mitosis, as is the case for $\operatorname{HIV}(4,5)$, and the decreased transduction of stationary-phase cells could be due to a block in the HFV life cycle after nuclear entry. The high transduction rate in stationary-phase cultures obtained with the G418 resistance assay indicates that HFV vectors efficiently enter non-proliferating cells and that functional vector genomes survive at least until these cultures are stimulated to divide the day after infection.

Potential in gene transfer applications. Vectors based on MLV and adeno-associated virus are the only integrating vector systems currently available, and both are limited by genome size, host range, and cell proliferation requirements $(27,33$, 43). The potential advantages of foamy virus vectors include a larger genome size, wide host range, and improved transduction of nondividing cells. HFV vectors are not inactivated by human serum and do not require the addition of polycations such as Polybrene for efficient transduction, so they may be especially well suited for in vivo gene delivery. Because Bel1 is required for high-level transcription from the viral LTR, integrated Bel1-deficient vectors should not activate downstream cellular genes by promoter insertion. These properties, as well as the lack of pathogenicity associated with foamy virus infections, suggest that foamy virus vectors may be safe and effective in gene therapy protocols.

\section{ACKNOWLEDGMENTS}

We thank R. Flügel for supplying pHSRV13, S. Yu for FAB cells, I. Alexander for helpful suggestions, and J. Alfano and R. Hirata for technical assistance.

This work was supported by grants HL53750, HL03100, and DK47754 from the U.S. National Institutes of Health (NIH) and grants from the American Society of Hematology and the American Philosophical Society.

\section{REFERENCES}

1. Achong, B. G., P. W. A. Mansel, M. A. Epstein, and P. Clifford. 1971. An unusual virus in cultures from a human nasopharyngeal carcinoma. J. Natl. Cancer Inst. 46:299-302.

2. Banapour, B., J. Sernatinger, and J. A. Levy. 1986. The AIDS-associated retrovirus is not sensitive to lysis or inactivation by human serum. Virology 152:268-271

3. Brown, P., G. Nemo, and D. C. Gajdusek. 1978. Human foamy virus: further characterization, seroepidemiology, and relationship to chimpanzee foamy viruses. J. Infect. Dis. 137:421-427.

4. Bukrinsky, M. I., S. Haggerty, M. P. Dempsey, N. Sharova, A. Adzhubel, L. Spitz, P. Lewis, D. Goldfarb, M. Emerman, and M. Stevenson. 1993. A nuclear localization signal within HIV-1 matrix protein that governs infection of non-dividing cells. Nature (London) 365:666-669.

5. Bukrinsky, M. I., N. Sharova, M. P. Dempsey, T. L. Stanwick, A. G. Bukrinskaya, S. Haggerty, and M. Stevenson. 1992. Active nuclear import of human immunodeficiency virus type 1 preintegration complexes. Proc. Natl. Acad. Sci. USA 89:6580-6584.

6. Duc-Ngugen, H., E. N. Rosenblum, and R. F. Zeigel. 1966. Persistent infection of a rat kidney cell line with Rauscher murine leukemia virus. J. Bacteriol. 92:1133-1140.

7. Fields-Berry, S. C., A. L. Halliday, and C. L. Cepko. 1992. A recombinant retrovirus encoding alkaline phosphatase confirms clonal boundary assignment in lineage analysis of murine retina. Proc. Natl. Acad. Sci. USA 89: 693-697.

8. Fischinger, P. J., S. Nomura, and D. P. Bolognesi. 1975. A novel murine oncornavirus with dual eco- and xenotropic properties. Proc. Natl. Acad. Sci. USA 72:5150-5155.

9. Flügel, R. M. 1991. Spumaviruses: a group of complex retroviruses. J. Acquired Immune Defic. Syndr. 4:739-750.

10. Flügel, R. M., A. Rethwilm, B. Maurer, and G. Darai. 1987. Nucleotide sequence analysis of the env gene and its flanking regions of the human spumaretrovirus reveals two novel genes. EMBO J. 6:2077-2084.

11. Graham, F. L., J. Smiley, W. C. Russell, and R. Nairn. 1977. Characteristics of a human cell line transformed by DNA from human adenovirus type 5. J. Gen. Virol. 36:59-72.
12. Haase, A. T., L. Stowring, J. D. Harris, B. Traynor, P. Ventura, R. Peluso, and M. Brahic. 1982. Visna DNA synthesis and the tempo of infection in vitro. Virology 119:399-410.

13. Harel, J., E. Rassart, and P. Jolicoeur. 1981. Cell cycle dependence of synthesis of unintegrated viral DNA in mouse cells newly infected with murine leukemia virus. Virology 110:202-207.

14. Hooks, J. J., and C. J. Gibbs, Jr. 1975. The foamy viruses. Bacteriol. Rev. 39:169-185.

15. Hoshino, H., H. Tanaka, M. Miwa, and H. Okada. 1984. Human T-cell leukemia virus is not lysed by human serum. Nature (London) 310:324-325.

16. Kao, F. T., and T. T. Puck. 1968. Genetics of somatic mammalian cells. VII. Induction and isolation of nutritional mutants in Chinese hamster cells. Proc. Natl. Acad. Sci. USA 60:1275-1281.

17. Keller, A., K. M. Partin, M. Löchelt, H. Bannert, R. M. Flügel, and B. R. Cullen. 1991. Characterization of the transcriptional trans activator of human foamy retrovirus. J. Virol. 65:2589-2594.

18. Kupiec, J.-J., J. Tobaly-Tapiero, M. Canivet, M. Santillana-Hayat, R. M. Flügel, J. Périès, and R. Emanoil-Ravier. 1988. Evidence for a gapped linear duplex DNA intermediate in the replicative cycle of human and simian spumaviruses. Nucleic Acids Res. 16:9557-9565.

19. Lewis, P., M. Hensel, and M. Emerman. 1992. Human immunodeficiency virus infection of cells arrested in the cell cycle. EMBO J. 11:3053-3058.

20. Löchelt, M., R. M. Flügel, and M. Aboud. 1994. The human foamy virus internal promoter directs the expression of the functional Bel 1 transactivator and Bet protein early after infection. J. Virol. 68:638-645.

21. Löchelt, M., W. Muranyi, and R. M. Flügel. 1993. Human foamy virus genome possesses an internal, Bel-1-dependent and functional promoter. Proc. Natl. Acad. Sci. USA 90:7317-7321.

22. Löchelt, M., H. Zentgraf, and R. M. Flügel. 1991. Construction of an infectious DNA clone of the full-length human spumaretrovirus genome and mutagenesis of the bel1 gene. Virology 184:43-54.

23. Loh, P. C., and K. S. Ang. 1981. Replication of human syncytium-forming virus in human cells: effect of certain biological factors and selective chemicals. J. Med. Virol. 7:67-73.

24. Macpherson, I., and M. Stoker. 1962. Polyoma transformation of hamster cell clones-an investigation of genetic factors affecting cell competence. Virology 16:147-151.

25. Maurer, B., H. Bannert, G. Darai, and R. M. Flügel. 1988. Analysis of the primary structure of the long terminal repeat and the gag and pol genes of the human spumaretrovirus. J. Virol. 62:1590-1597.

26. Mergia, A., and P. A. Luciw. 1991. Replication and regulation of primate foamy viruses. Virology 184:475-482.

27. Miller, A. D. 1992. Retroviral vectors. Curr. Top. Microbiol. Immunol. 158: $1-24$.

28. Miller, A. D., and C. Buttimore. 1986. Redesign of retrovirus packaging cell lines to avoid recombination leading to helper virus production. Mol. Cell. Biol. 6:2895-2902.

29. Miller, A. D., and G. J. Rosman. 1989. Improved retroviral vectors for gene transfer and expression. Biotechniques 7:980-990.

30. Miller, D. G., M. A. Adam, and A. D. Miller. 1990. Gene transfer by retrovirus vectors occurs only in cells that are actively replicating at the time of infection. Mol. Cell. Biol. 10:4239-4242.

31. Miller, D. G., R. H. Edwards, and A. D. Miller. 1994. Cloning of the cellular receptor for amphotropic murine retroviruses reveals homology to that for gibbon ape leukemia virus. Proc. Natl. Acad. Sci. USA 91:78-82.

32. Muranyi, W., and R. M. Flügel. 1991. Analysis of splicing patterns of human spumaretrovirus by polymerase chain reaction reveals complex RNA structures. J. Virol. 65:727-735

33. Muzyczka, N. 1992. Use of adeno-associated virus as a general transduction vector for mammalian cells. Curr. Top. Microbiol. Immunol. 158:97-129.

34. Palmer, T. D., R. A. Hock, W. R. A. Osborne, and A. D. Miller. 1987. Efficient retrovirus-mediated transfer and expression of a human adenosine deaminase gene in diploid skin fibroblasts from an adenosine deaminase-deficient human. Proc. Natl. Acad. Sci. USA 84:1055-1059.

35. Parks, W. F., and G. J. Todaro. 1972. Biological properties of syncytiumforming ("foamy") viruses. Virology 47:673-683.

36. Parks, W. F., G. J. Todaro, E. M. Scolnick, and S. A. Aaronson. 1971 RNA-dependent DNA polymerase in primate syncytium-forming (foamy) viruses. Nature (London) 229:258-260.

37. Quade, K. 1979. Transformation of mammalian cells by avian myelocytomatosis virus and avian erythroblastosis virus. Virology 98:461-465.

38. Renshaw, R. W., M. A. Gonda, and J. W. Casey. 1991. Structure and transcriptional status of bovine syncytial virus in cytopathic infections. Gene 105:179-184.

39. Rethwilm, A., G. Baunach, K.-O. Netzer, B. Maurer, B. Borisch, and V. ter Meulen. 1990. Infectious DNA of the human spumaretrovirus. Nucleic Acids Res. 18:733-738.

40. Rethwilm, A., O. Erlwein, G. Baunach, B. Maurer, and V. ter Meulen. 1991. The transcriptional transactivator of human foamy virus maps to the bel1 genomic region. Proc. Natl. Acad. Sci. USA 88:941-945.

41. Roe, T. Y., T. C. Reynolds, G. Yu, and P. Brown. 1993. Integration of murine leukemia virus DNA depends on mitosis. EMBO J. 12:2099-2108. 
42. Russell, D. W., M. S. Berger, and A. D. Miller. 1995. The effects of human serum and cerebrospinal fluid on retroviral vectors and packaging cell lines. Human Gene Ther. 6:635-641.

43. Russell, D. W., A. D. Miller, and I. E. Alexander. 1994. Adeno-associated virus vectors preferentially transduce cells in S phase. Proc. Natl. Acad. Sci. USA 91:8915-8919.

44. Schliephake, A. W., and A. Rethwilm. 1994. Nuclear localization of foamy virus Gag precursor protein. J. Virol. 68:4946-4954.

45. Schmidt, M., and A. Rethwilm. 1995. Replicating foamy virus-based vectors directing high level expression of foreign genes. Virology 210:167-178.

46. Schweizer, M., U. Fleps, A. Jäckle, R. Renne, R. Turek, and D. NeumannHaefelin. 1993. Simian foamy virus type 3 (SFV-3) in latently infected Vero cells: reactivation by demethylation of proviral DNA. Virology 192:663-666.

47. Schweizer, M., R. Turek, H. Hahn, A. Schliephake, K.-O. Netzer, G. Eder, M. Reinhardt, A. Rethwilm, and D. Neumann-Haefelin. 1995. Markers of foamy virus infections in monkeys, apes, and accidentally infected humans: appropriate testing fails to confirm suspected foamy virus prevalence in humans. AIDS Res. Hum. Retroviruses 11:161-170.

48. Takeuchi, Y., F.-L. C. Cosset, P. J. Lachmann, H. Okada, R. A. Weiss, and
M. K. L. Collins. 1994. Type C retrovirus inactivation by human complement is determined by both the viral genome and the producer cell. J. Virol. 68:8001-8007.

49. Venkatesh, L. K., P. A. Theodorakis, and G. Chinnadurai. 1991. Distinct cis-acting regions in U3 regulate trans-activation of the human spumaretrovirus long terminal repeat by the viral bel1 gene product. Nucleic Acids Res. 19:3661-3666.

50. Wei, C. M., M. Gibson, P. G. Spear, and E. M. Scolnick. 1981. Construction and isolation of a transmissible retrovirus containing the $s r c$ gene of Harvey murine sarcoma virus and the thymidine kinase gene of herpes simplex virus type 1. J. Virol. 39:935-944.

51. Weiss, R. A., N. Teich, H. E. Varmus, and J. M. Coffin. 1984. RNA tumor viruses, 2nd ed. Cold Spring Harbor Laboratory, Cold Spring Harbor, N.Y.

52. Welsh, R. M., N. R. Cooper, F. C. Jensen, and M. B. A. Oldstone. 1975. Human serum lyses RNA tumour viruses. Nature (London) 257:612-614.

53. Yu, S. F., and M. L. Linial. 1993. Analysis of the role of the bel and bet open reading frames of human foamy virus by using a new quantitative assay. J. Virol. 67:6618-6624. 